\title{
Using CRISPR-Cas9 for Therapeutic Protein Production (Review Article)
}

\author{
Ashraf Ullah Khan ${ }^{1}$, Zabi Ullah ${ }^{2}$ \\ ${ }^{1}$ Faculty of Pharmacy and Health Sciences, University of Balochistan (Main Branch) \\ Quetta, Pakistan. \\ Email: ashrafstanikzai [AT] gmail.com \\ ${ }^{2}$ Faculty of Curative Medicine, Malalay Institute of Higher Education \\ Aino Maina, Kandahar, Afghanistan \\ Email: zabiullahkhanstanikzai2015 [AT] yahoo.com
}

\begin{abstract}
Existence of CRISPR/Cas9 systems in bacteria and archaea has been noted to be the reason for these organisms' ability to disarm invading nucleic acids. Such immunity is noted to arise from the targeting of the invading nucleic acids by guiding RNAs (sgRNAs), their cleavage by Cas9 (an endonuclease), and their subsequent integration into CRISPR locus. Recent studies have shown that the CRISPR/Cas9 tool can be adopted for gene editing in eukaryotic cells and thus offering potential for its use to treat genetic conditions. In this review, CRISPR/Cas9 has been shown to be an effective genome-editing tool with studies showing efficacy in zygote editing, in-vivo editing of somatic cells and ex-vivo editing of somatic cells. Occurrence of off-target effects however make zygote editing in human cells ethically questionable due to possibility of introducing unwanted mutations that may be passed on to the progeny. Nevertheless, observations that such off-target effects arise mainly from the promiscuity of sgRNAs rather that errors in CRISPR/Cas9 system show promise for increased specificity by developing better sgRNAs. Such increased specificity will facilitate the adoption of CRISPR/Cas9 for clinical use in treatment of conditions such as $\beta$-thalassemia, cystic fibrosis, Duchenne muscular dystrophy and HIV.
\end{abstract}

Keywords-CRISPR/Cas9, genome editing, gene therapy, sgRNA

\section{USING CRISPR-CAS9 FOR THERAPEUTIC PROTEIN PRODUCTION}

Evidence of a genetic etiology for various illnesses has increased efforts to find remedies that target the defective genes. Specifically, with the completion of the human genome project, scientist have been able to map out the loci of genes implicated in various ailments such as cancers and thus potentiating the use of genetic engineering technologies to edit such defective genes. One of these genome-editing technologies is the CRISPR/Cas9 system, a customizable approach to edit DNA based on the ability of the endonuclease Cas9 to bind and cleave specific nucleotide sequences in the human genome [1]. CRISPR/Cas systems are systems naturally developed in bacteria and archaea that confer immunity to these organisms against invading plasmids and viruses [2]. The adaptive immunity provided by these systems in the prokaryotes arises from the ability of CRISPR RNAs (crRNAs) to target the invading nucleic acids thus leading to the silencing of these invading nucleic acids through their cleavage and integration of the resultant fragments into the CRISPR locus [3]. CRISPR is the acronym for "clustered regularly interspaced short palindromic repeats" [3], a reference to the genomic locus of such repeating nucleotide sequences in bacterial and archaea [1]. Cas9 stands for "CRISPR-associated protein 9" [4], a CRISPR-associated endonuclease that is involved in the crCRNA-guided inactivation of foreign nucleic acids [3]. In 2012, Jinek et al. showed that it was possible to recruit Cas9 to specific genome loci through standard base pairing. In their study, Jenik et al. (2012) fused crRNA to the trans-activating crRNA (tracrRNA) of the Streptococcus pyogenes to generate a single guide RNA (sgRNA). The sgRNA was able to recruit Cas9 at specific genome loci and thus facilitating cleavage of sequences in these loci to achieve a desired effect [3]. This technology has been adopted in the development of therapeutic proteins that help in genome editing to inactivate mutated sequences that are linked to genetic diseases. This paper reviews such use of CRISPR-Cas9 to develop therapeutic proteins for various illnesses.

\section{APPLICATIONS OF CRISPR-CAS9 FOR THERAPEUTIC PURPOSES}

Most of the studies published on use of CRISPR-Cas9 for therapeutic purposes relate to laboratory experiments with prokaryotes, eukaryotic cells (including humans), and experimental animals. However, the CRISPR-Cas9 technology is yet to be adopted in clinical use as evident from the lack of any clinical trials using the technology (based on search of trials for CRISPR-Cas9, CRISPR, Cas9 at www. clinicaltrials.gov/). Such failure to use the technology in clinical trials may be explained by the ethical issues accompanying genome editing in humans and uncertainty on the side effects of offtarget genome changes that may arise with use of genome-editing tools [5]. Specifically, editing of germ-line cells poses a 
significant ethical dilemma since any genetic errors introduced in such cells can be passed on to subsequent generations and thus possibly leading to high prevalence of unforeseen genetic illnesses in the population [5]. Such concerns have limited the adoption of genome editing therapy in humans. For example, the first gene-editing case in people was done only recently, in 2014, using Zinc finger nucleases (SFNs) that target specific DNA sequences, to treat people with HIV [6]. Even then, such treatment has mainly entailed ex-vivo strategies, where gene edited cells are introduced into the patients' bodies rather than in-vivo strategies, where the gene-editing tools are administered themselves instead of the altered cells using viral or non-viral vectors [4]. For CRISPR/Cas9 system, the most recent advance in their use in humanassociated cells has been the February 2016 approval for U.K. scientists to use the technology to edit human embryos for a duration of seven days, after which the embryos are to be destroyed [7]. The subsequent review of the therapeutic use of CRISPR-Cas9 system is thus based on laboratory data instead of human clinical trials.

\section{USE OF CRISPR/CAS9 FOR ZYGOTE EDITING}

Use of CRISPR/Cas9 system to edit zygotes has been successfully tried in mice studies. In this approach, components of the CRISPR/Cas9 tool (e.g. Cas9 messenger RNA, sgRNA, and Homology Directed Repair (HDR) template; a strategy that seeks to correct errors in double-stranded DNA, are injected into the zygote or into an early-stage embryo [4]. In one such study, Wang et al. (2013) used the CRISPR/Cas9 tool to introduce mutations in mice through the co-injection of Cas9 mRNA and sgRNAs into mouse embryonic stem cells. Using this strategy, Wang et al. (2013) were able to target Tet1 and Tet 2 genes leading to the production of mice biallelic mutations at an efficiency rate of $80 \%$ [8]. Further, when the researchers integrated mutant oligonucleotides into the sgRNAs that were co-injected with Cas 9 mRNAs, they were able to induce point mutations in the two target genes in the resultant mice. This study demonstrated that the CRISPR/Cas 9 editing tool could be used to generate mutations, which may be useful in evaluation of genes that are functionally redundant or in the evaluation of genes that have epistatic interactions. However, the study does not show whether CRISPR/Cas9 could be used to correct existing genetic disorders, a subject that was addressed in a different study reviewed subsequently [8].

In a different study, Wu et al. (2013) showed that the CRISPR/Cas9 editing tool could be used to correct mutations that led to cataracts in mice. In the study, Wu et al. (2013) co-injected zygotes of such mice with Cas9 mRNA and sgRNAs that targeted the Crygc gene. The study showed that the Cas 9 system was able to repair the mutation in the Cryg $c$ gene based on HDR template oligonucleotides supplied by the researchers exogenously or based on endogenous wild-type-allele template [9]. The correction was noted to occur efficiently with limited off-target modifications, with the resultant mice retaining their fertility and successfully passing on the corrected allele to their offspring [9]. The results of this study thus showed that CRISPR/Cas9 was not only useful in generating mutations but also in correcting disease-causing mutations.

Before the U.K. approval of a CRISPR/Cas9-based study using human embryo cells, an earlier study that had employed the technology on human embryo cells had resulted into divisions among scientists over the ethics of using such technology in embryo cells $[5,10]$. In this earlier study, Liang et al. (2015) had used CRISPR/Cas9 to cleave endogenous $\beta$-globin gene (HBB, a gene implicated in causing $\beta$-thalassemia, a blood disorder) using Cas9 mRNA and sgRNA transfected into human triponuclear (3PN) zygotes. Nevertheless, the study found that CRISPR/Cas9 tool also resulted in high off-target effects (cleavage at non-targeted loci) and could introduce unwanted mutations due to the competition between exogenously supplied oligonucleotide template and the endogenous delta-globin gene [11]. Such results indicated that CRISPR/Cas9 may have low fidelity and specificity, a finding that led the researchers to recommend the non-use of CRISPR/Cas9 for clinical applications [11]. In summary, therefore, while the efficiency of CRISPR/Cas9 in mice embryos has been shown to be high with low off target effects, more research on the off target effects noted in human cells is needed before the technology can be reliably used in clinical applications targeting human embryo cells. Further, due to the possibility of use of CRISPR/Cas9 for non-medical purposes where embryos are involved such as altering traits of resultant children [4], use of CRISPR/Cas9 technology to edit human zygotes poses a grave ethical dilemma.

\section{IN-VIVO EDITING OF SOMATIC CELLS USING CRISPR/CAS9 TECHNOLOGY}

While the editing of germ-line cells poses significant issues due to the possibility of transmitting any generated mutations to the progeny [5], editing of somatic cells poses a significantly lesser issue since any mutations arising from the process are not transmitted to the offspring. Such lower risk may explain the performance of in-vivo CRISPR/Cas9 tests targeting somatic cells, albeit in experimental animals. In one such study, Yin et al. (2014) showed that it was possible to use CRISP/Cas9 mechanism to correct for hereditary tyrosinemia type I in mouse models that had been developed to express this disease [12]. The disease arises from the mutation in the gene encoding for fumarylacetoacetate hydrolase (FAH), an enzyme that catalyzes the transformation of fumarylacetoacetate to form fumarate in the catabolism process for tyrosine and phenylalanine in hepatocytes [13]. In the study by Yin et al. (2014), the authors targeted the Fah gene using a pX330 vector into which three sgRNAs that targeted Fah had been cloned. Further, they synthesized a 199-nucleotide single stranded DNA (ssDNA) that would facilitate homologous recombination and ensure the correction of the $\mathrm{G} \rightarrow \mathrm{A}$ splicing mutation that is usually implicated in tyrosinemia I. This ssDNA had a wild-type G nucleotide and was designed to flank the sgRNA target region [12]. The researchers evaluated the efficacy of the CRISPR/Cas9 system in correcting tyrosinemia I by having controls that would highlight whether observations were a result of the CRISPR/Cas9 effect or 
other confounding factors. All the mice used for the study had the Fah mutation. In the first group of mice, they injected saline via the tail veins, while the second group of mice was given the ssDNA oligonucleotide only via the same route [12]. In the third group, the ssDNa oligonucleotides and the vector expressing only Cas9 mRNA (i.e., unguided Cas9) were administered whereas in the fourth group, SSDNA oligonucleotides, vector expressing Cas9 mRNA and a guiding sgRNA were administered [12]. The results of the study indicated that apart from the fourth group, the mice in the other three groups that did not receive NTBC-containing water quickly lost about $20 \%$ of their body weight and thus had to be euthanized [12]. Mice in the fourth group either did not lose weight or lost a lower proportion of weight based on the guiding sgRNA used. Moreover, the mice in this group that lost weight were able to regain the lost weight on provision of NTBC water for 7 days, even with subsequent withdrawal of the NTBC water for 28 days [12]. Analysis of liver damage in the fourth group also showed that these mice had significantly lower damage and immunohistochemical staining using a Fah-staining antibody showed that the CRISPR/Cas9 gene editing was successful in generating Fah ${ }^{+}$hepatocytes within 30 days of treatment [12]. The genome-editing in this study occurred with low off-target effects $(<0.3 \%$ where FAH2 $\operatorname{sgRNAs}$ were used), and though the repair frequency was also low $(0.4 \% \pm 0.12 \%)$ [12], positive selection of the repaired hepatocytes showed that CRISPR/Cas9 gene editing may offer an important tool for treatment of tyrosinemia type I.

In a different study, Ding et al. (2014) also showed the efficacy of using CRISPR/Cas9 to edit proprotein convertase subtilisin/kexin type 9 (PCSK9) through an in-vivo approach. Mutations in PCSK9 are associated with a reduction in the levels of low-density lipoprotein (LDLP) and thus lowering the risk of cardiovascular disease [14]. Using CRISPR/Cas9 tool, the authors found out that it was possible to introduce mutations in Pcsk9 in mice liver thus reducing the encoding of the PCSK9 protein. In the study, the authors did not detect any off-target effects, and mutagenesis in Pcsk 9 was noted to be more than $50 \%$ in some cases, thus leading to significant drop in plasma PCSK9, increase in LDL receptor levels in the liver, and decrease in plasma cholesterol levels [14]. Such results indicated efficiency of the CRISPR/ Cas9 in disruption of gene function, which, in this case, may have therapeutic applications in reducing the risk of cardiovascular disease.

Lin et al. (2014) have also demonstrated the efficacy of CRISPR/Cas9 system in editing in-vivo. In the study, the authors used an HBV-vector transfected to express Cas9 mRNAs and sgRNAs targeting various loci of the HBV-genome in mice modeled to express chronic HBV infection in their livers. Intravenous administration of the vector containing the CRISPR/Cas9 components to the mice led to the cleavage of the vector and subsequent reduction in surface antigen for hepatitis B in the serum [15]. While the mice used did not produce the closed circular DNA (cccDNA) that helps the HB virus to avoid complete elimination in the liver, the authors were able to verify the efficacy of the CRISPR/Cas9 system in eliminating HBV using a human cell line that was engineered to express duck HBV [15]. However, the possibility of residual viral DNA as highlighted in the human cell line experiments necessitates the development of more efficient delivery options before the clinical application of the technology since such residual viral infections may renew the HBV infection $[4,15]$.

\section{EX-VIVO EDITING OF SOMATIC CELLS USING CRISPR/CAS9 SYSTEM}

Ex-vivo editing of somatic cells may provide advantages over in-vivo editing due to ability to monitor progress before administration of edited cells, but it also presents challenges of culturing stem/progenitor cells derived from the patient that need to be gene-edited [4]. Recent progress enabling the generation of induced pluripotent stem cells (iPSCs) has however provided promise for ex-vivo gene editing in humans since such iPSCs can be propagated and differentiated to form any type of body cell [4]. Such iPSCs have been used to prove the application of CRISPR/ Cas9 tool for ex-vivo gene editing in various studies.

In one of the first studies using iPSCs for CRISPR/ Cas9 evaluation, Xie et al. (2014) were able to demonstrate efficacy of CRISPR/ Cas9 in treating $\beta$-thalassemia through an ex-vivo gene-editing approach. In the study, the researchers generated iPSCs from fibroblasts of a patient who had homozygous alleles for $\beta$-thalassemia [16]. The generated iPSCs were then transfected with vectors containing CRISPR/Cas9 components targeting the $H B B$ gene mutations associated with causing the disease and an HDR DNA template. The study findings showed that CRISPR/Cas9 was able to correct the mutations in iPSCs without having any off-target effects, with the corrected iPSCs showing full pluripotency and normal karyotypes [16]. Differentiation of the corrected iPSCs into erythrocytes using a monolayer culture technology showed that the corrected iPSCs helped restore the expression of HBB when compared to uncorrected iPSCs [16]. This study showed that CRISPR/Cas9 technology could be used successfully to generate functional somatic cells that can then be administered in transplantations to correct genetic-based ailments.

A study by Schwank et al. (2013), the researchers were also able to demonstrate the use of CRISPR/Cas9 system to correct mutations that hinder cAMP-mediated swelling of epithelial organoids in cystic fibrosis. Using intestinal organoids, the researchers were able to correct the cystic fibrosis-causing mutation in the gene encoding for proteins of the cystic fibrosis transmembrane conductance regulator [17]. CFTR is an ion channel whose regulation of ion concentration across the cell membrane facilitates the transport of epithelial fluid. A dysfunctional CFTR leads to mucus accumulation in the pulmonary tract and gastrointestinal tract leading to problems such as breathing difficulties [4]. In the study by Schwank et al. (2013), the researchers isolated intestinal stem cells from patients suffering from cystic fibrosis, cultured them to generate $3 \mathrm{D}$ organoid cultures, and transfected the resultant organoids with vectors cloned with CRISPR/Cas9 components 
targeting CFTR locus [17]. The study showed effective correction of the CFTR mutations with only few off-target effects thus highlighting the potential use of CRISPR/ Cas9 for ex-vivo treatment of cystic fibrosis.

Two other studies have shown the potential for use of CRISPR/Cas9 system to correct mutations responsible for Duchenne muscular dystrophy (DMD) using ex-vivo approaches. In one of the studies, Li et al (2015) used iPSCs derived from patients with DMD to demonstrate the use of CRISPR/Cas9 and TALEN, another gene-editing technology. In the study, $\mathrm{Li}$ et al. (2015) were able to regenerate a full-length dystrophin gene on transfecting the iPSCs with a vector containing the CRISPR/Cas9 components and HDR DNA template. Differentiation of the corrected iPSCs into skeletal muscle cells showed normal expression of the wild-type dystrophin protein [18], thus indicating the success of the technology in correcting mutations responsible for causing DMD. The second study, which was conducted by Ousterout et al. (2015), showed that it was possible to use the CRISPR/Cas9 system to restore the reading frame that restores the functionality of the dystrophin gene. The researchers used myoblasts derived from patients suffering from DMD, which were then transfected with a vector containing the CRISPR/Cas9 components targeting mutation prone exons for deletion [19]. Using this technology, the researchers were able to delete exons 45-55, which contribute up to $60 \%$ of the mutations implicated in causing DMD [19]. Deletion of these exons helped restore the expression and function of the dystrophin gene as was shown in in vitro assays using the corrected myoblasts and in in-vivo assays on transplantation of the corrected myoblasts into mutant mice [19]. Overall, these ex-vivo studies prove the applicability of CRIPR/Cas9 technology in editing defective genes. Clinical application may however take some time due to ambiguity on specificity of the technology as discussed subsequently.

\section{CHALLENGES IN THE CLINICAL APPLICATION OF CRISPR/CAS9 TECHNOLOGY}

The principal challenge in the adoption of CRISPR/Cas9 technology relates to its safety, which in turn is influenced by its specificity. High occurrence of off-target effects may be hazardous since, as Lanphier and Urnov (2015) noted, they may lead to mutations with multiple adverse effects that outweigh the benefits. Specifically, since editing of embryonic cells would confer changes that would be transmitted to the progeny [5], use of CRISPR/Cas9 in editing embryonic cells for clinical applications is not advisable, especially where alternative safer technologies such as in-vitro fertilization are available [4].

Ambiguity in research on the specificity of CRISPR/Cas9 technology also poses challenges in its adoption for clinical use. As noted in a review by O'Green, Yu and Segal (2015) multiple studies have provided mixed results on the specificity of CRISPR/Cas9 technology with some showing low off-target effects (high specificity), while others show high off-target effects (low specificity). Nevertheless, advances in technologies for detecting off-target effects have helped to clarify specificity of CRISPR/Cas9 technology. For instance, these advances have highlighted that differences in CRISPR/Cas9 specificity do not mainly arise from the technology itself but from the promiscuity noted of sgRNAs [20]. As such, the challenge for increasing specificity of CRISPR/Cas9 has turned to the accuracy in prediction of the sgRNA that would confer high on-target activity without significant off-target effects [20]. With increasing knowledge of the loci of various genes following the completion of the human genome project, promise for developing sgRNAs that have lower off-target effects exist. Such capability to improve the specificity of CRISPR/ Cas9 specificity by focusing on bettering sgRNAs used makes CRISPR/Cas9 technology a better gene-editing tool compared to other existing tools such as ZFN and TALEN, which require more effort for assembly of proteins that can help in improving their specificity [20].

\section{CONCLUSION}

CRISPR/Cas9 tool offers a novel technology for therapeutic applications that seek to reverse conditions that arise with defective gene function. While not currently used in clinical applications, CRISPR/Cas9 has shown promise for effective gene editing to correct for mutations implicated in various genetic diseases. Current tests of the technology have involved zygote-editing experiments, in-vivo editing of genes in experimental animals developed to express diseases of interest, and ex-vivo editing of defective genes followed by the transplantation of the corrected cells into experimental animals. A core challenge in all these tests has been the occurrence of off-target effects in some of the studies that indicates potential for the technology to also introduce unwanted mutations apart from correcting the mutations of interest. Nevertheless, analysis of the off-target effects showing that such effects arise from promiscuity of sgRNAs rather than defects in CRISPR/Cas9 per se highlight that CRISPR/Cas9 may be a clinically important tool for gene editing in future when better methods for enhancing the specificity of sgRNAs are developed.

\section{REFERENCES}

[1] Genome editing for all. (2014). Nature Biotechnology, 32(4), 295. doi:10.1038/nbt.2879

[2] Lee, J. S., Grav, L. M., Lewis, N. E., \& Kildegaard, H. F. (2015). CRISPR/ Cas9-mediated genome engineering of $\mathrm{CHO}$ cell factories: Applications and perspectives. Biotechnology Journal, 10(7), 979-94. doi:10.1002/biot.201500080032

[3] Jinek, M., Chylinski, K., Fonfara, I., Hauer, M., Doudna, J. A., \& Charpentier, E. (2012). A programmable dual-RNAguided DNA endonuclease in adaptive bacterial immunity. Science, 337, 816-821. doi:10.1126/science.1225829 
[4] Savić, N., \& Schwank, G. (2016). Advances in the therapeutic CRISPR/Cas9 genome editing. Translational Research, 168(), 15-21. http://dx.doi.org/10.1016/j.trsl.2015.09.008

[5] Lanphier, E., \& Urnov, F. (2015). Don't edit the human germ line. Nature, 519, 410-411. Retrieved from http://www.nature.com/polopoly_fs/1.17111!/menu/main/topColumns/topLeftColumn/pdf/519410a.pdf

[6] Reardon, S. (2015). Gene-editing wave hits clinic. Nature, 527, 146-147. Retrieved from http://www.nature.com/polopoly_fs/1.18737!/menu/main/topColumns/topLeftColumn/pdf/nature.2015.18737.pdf

[7] Callaway, E. (2016). Embryo editing gets green light. Nature, 530, 18 . Retrieved from http://www.nature.com/polopoly_fs/1.19270!/menu/main/topColumns/topLeftColumn/pdf/nature.2016.19270.pdf

[8] Wang, H., Yang, H., Shivalila, C. S., Dawlaty, M. M., Cheng, A. W., Zhang, F., \& Jaenisch, R. (2013). One-step generation of mice carrying mutations in multiple genes by CRISPR/Cas-mediated genome engineering. Cell, 153(4), 910-918. doi:10.1016/j.cell.2013.04.025.

[9] Wu, Y., Liang, D., Wang, Y., Bai, M., Tang, W., Bao, S., ... Li, J. (2013). Correction of a genetic disease in muse via use of CRISPR-Cas9. Cell Stem Cell, 13(6), 659-662. http://dx.doi.org/10.1016/j.stem.2013.10.016

[10] Cyranoski, D. (2015). Embryo editing divides scientists. Nature, 519, 272. Retrieved from http://www.nature.com/polopoly_fs/1.17131!/menu/main/topColumns/topLeftColumn/pdf/519272a.pdf

[11] Liang, P., Xu, Y., Zhang, X., Ding, C.,Huang, R., Zhang, Z., ... Huang, J. (2015). CRISPR/Cas9-mediated gene editing in human triponuclear zygotes. Protein Cell, 6(5), 363-372. doi:10.1007/s13238-015-0153-5

[12] Yin, H., Xue, W., Chen, S., Bogorad, R., Benedetti, E., Grompe, M., Koteliansky, V., ... Anderson, D. G. (2014) Genome editing with Cas9 in adult mice corrects a disease mutation and phenotype. Nature Biotechnology, 32(6), 5513. doi:10.1038/nbt.2884

[13] Nelson, D. L., \& Cox, M. M. (2004). Lehninger principles of biochemistry (4th ed.). New York: W. H. Freeman \& Company

[14] Ding, Q., Strong, A., Patel, K. M., Ng, S-L., Gosis, B. S., Regan, S. N., .. Musunuru, K. (2014). Permanent alteration of PCSK9 with in vivo CRISPR-Cas9 genome editing. Circulation Research, 115(5), 488-492, doi:10.1161/CIRCRESAHA.115.304351

[15] Lin, S. R., Yang, H. C., Kuo, Y. T., Liu, C. J., Yang, T. Y., Sung, K. C., .. Chen, P. J. (2014). Molecular Therapy. Nucleic Acids, 19(3), e186. doi:10.1038/mtna.2014.38.

[16] Xie, F., Ye, L., Chang, J. C., Beyer, A. I., Wang, J., ... Kan, Y. W. (2014). Seamless gene correction of $\beta$-thalassemia mutations in patient-specific iPSCs using CRISPR/Cas9 and piggyBac. Genome Research, 24(9), 1526-33, doi:10.1101/gr.173427.114.

[17] Schwank, G., Koo, B. K., Sasselli, V., Dekkers, J. F., Heo, I., Demircan, T., Clevers, H. (2013). Functional repair of CFTR by CRISPR/Cas9 in intestinal stem cell organoids of cystic fibrosis patients. Cell Stem Cell, 13(6), 653-8. doi:10.1016/j.stem.2013.11.002.

[18] Li, H. L., Fujimoto, N., Sasakawa, N., Shirai, S., Ohkame, T., Sakuma, T., ... Hotta, A. (2015). Precise correction of the dystrophin gene in duchenne muscular dystrophy patient induced pluripotent stem cells by TALEN and CRISPRCas9. Stem Cell Reports, 4(1):143-54. doi: 10.1016/j.stemcr.2014.10.013.

[19] Ousterout, D. G., Kabadi, A. M., Thakore, P. I., Majoros, W. H., Reddy, T. E., \& Gersbach, C. A. (2015). Multiplex CRISPR/Cas9-based genome editing for correction of dystrophin mutations that cause Duchenne muscular dystrophy. Nature Communications, 6, 6244. doi: 10.1038/ncomms 7244

[20] O'Green, H., Yu, A. S., \& Segal, D. J. (2015). How specific is CRISPR/Cas9 really? Current Opinion in Chemical Biology, 29, 72-78. http://dx.doi.org/10.1016/j.cbpa.2015.10.001 